\title{
BMJ Open Protocol for Meal-time Administration of Exenatide for Glycaemic Control in Type 1 Diabetes Cases (The MAG1C trial): a randomised, double-blinded, placebo-controlled trial
}

\author{
Nicklas Järvelä Johansen, ${ }^{1}$ Thomas Fremming Dejgaard, ${ }^{1}$ Asger Lund, ${ }^{1}$ \\ Tina Vilsbøll, ${ }^{1,2}$ Henrik Ullits Andersen, ${ }^{3}$ Filip Krag Knop ${ }^{1,2,4}$
}

To cite: Johansen NJ, Dejgaard TF, Lund A, et al. Protocol for Meal-time Administration of Exenatide for Glycaemic Control in Type 1 Diabetes Cases (The MAG1C trial): a randomised, double-blinded, placebocontrolled trial. BMJ Open 2018;8:e21861. doi:10.1136/ bmjopen-2018-021861

- Prepublication history for this paper is available online. To view these files, please visit the journal online (http://dx.doi org/10.1136/bmjopen-2018021861).

Received 22 January 2018 Revised 15 May 2018 Accepted 24 May 2018

\section{Check for updates}

${ }^{1}$ Steno Diabetes Center Copenhagen, Gentofte Hospital, Hellerup, Denmark

${ }^{2}$ Department of Clinical Medicine, University of Copenhagen, Copenhagen, Denmark

${ }^{3}$ Steno Diabetes Center Copenhagen, Gentofte, Denmark ${ }^{4}$ Novo Nordisk Foundation Center for Basic Metabolic Research, University of Copenhagen, Copenhagen, Denmark

Correspondence to Professor Filip Krag Knop; filipknop@dadlnet.dk

\section{ABSTRACT}

Introduction Persons with type 1 diabetes require intensive insulin therapy to achieve glycaemic control, but side effects, including hypoglycaemia and weight gain, may reduce treatment compliance. We hypothesise that add-on treatment of the short-acting glucagon-like peptide-1 receptor agonist, exenatide, to insulin therapy in persons with type 1 diabetes will reduce insulin requirements, glycaemic excursions and body weight and improve glycaemic control without increasing the risk of hypoglycaemia. The present article describes a protocol developed to test this hypothesis.

Methods and analysis One-hundred adult persons with type 1 diabetes for more than 1 year, insufficient glycaemic control (glycated haemoglobin A1c (HbA1c) between 58 and $86 \mathrm{mmol} / \mathrm{mol}$ ) and body mass index $>22.0 \mathrm{~kg} / \mathrm{m}^{2}$ will be randomised to either exenatide $10 \mu \mathrm{g}$ three times per day (at meal times) or placebo as add-on therapy to regular basal-bolus insulin treatment for 26 weeks. Primary endpoint is change in $\mathrm{HbA1}$ c between the two groups at end of treatment. Secondary endpoints include change in glycaemic excursions (assessed by continuous glucose monitoring); insulin dose; hypoglycaemic and adverse events; body weight, lean body and fat mass; dietary patterns; quality of life and treatment satisfaction; cardiovascular-disease risk profile; metabolomics; and arginine-tested plasma glucose, glucagon and C-peptide levels.

Ethics and dissemination The study is approved by the Danish Medicines Agency, the Regional Scientific Ethics Committee of the Capital Region of Denmark and the Data Protection Agency. The study will be carried out under the surveillance and guidance of the good clinical practice (GCP) unit at Copenhagen University Hospital Bispebjerg in accordance with the ICH-GCP guidelines and the Helsinki Declaration. Positive, negative as well as inconclusive results will be sought disseminated at scientific meetings and in international peer-reviewed scientific journals. Trial registration number NCT03017352.

\section{INTRODUCTION}

\section{Background and rationale}

Type 1 diabetes is a global disease affecting millions of people with increasing incidence. ${ }^{12}$

\section{Strengths and limitations of this study}

- First randomised, double-blinded, placebo-controlled trial to investigate a short-acting glucagon-like peptide-1 receptor agonist as add-on therapy in type 1 diabetes.

- This study will include normal to overweight persons with insufficient glycaemic control, who represent a large proportion of persons with type 1 diabetes worldwide.

- The study is powered to draw conclusions on glycaemic control (as assessed by glycated haemoglobin), glycaemic variability and time spent in near normoglycaemia.

- The study may not be sufficiently powered to draw firm conclusions on all secondary endpoints.

The majority of persons with type 1 diabetes do not achieve glycaemic control, and up to $50 \%$ are overweight or obese with a body mass index $>25 \mathrm{~kg} / \mathrm{m}^{2}{ }^{3-6}$ Intensive insulin treatment is necessary to ensure glycaemic control that delays the onset and slows the progression of microvascular complications, that is, diabetic retinopathy, neuropathy, nephropathy and macrovascular disease. ${ }^{7-9}$ Failure to achieve glycaemic control may occur due to side effects of intensive insulin treatment, that is, weight gain and hypoglycaemia. $^{1011}$ Both weight gain and hypoglycaemia have been shown to reduce treatment compliance. Severe hypoglycaemia is associated with serious physiological and psychological comorbidity and even death. ${ }^{11}$ Milder hypoglycaemic episodes lead to fear of future episodes, and unwanted weight gain leads to reduced insulin doses. ${ }^{12}{ }^{13}$ Overweight itself is unwanted among persons with type 1 diabetes and associated with its own problems, 
for example, hypertension, cancer and increased cardiovascular disease risk. ${ }^{14}$

To improve treatment of type 1 diabetes, these problems must always be considered and addressed. Add-on therapy of non-insulin drugs developed for type 2 diabetes has recently gained increasing interest within type-1-diabetes research. ${ }^{15}$ The incretin hormone, glucagon-like peptide-1 (GLP-1), regulates glucose metabolism through GLP-1 receptor-induced pancreatic and extrapancreatic effects, for example, increased glucose-dependent insulin secretion, lowered postprandial glucagon secretion and reduced rate of gastric emptying. ${ }^{16}$ Furthermore, GLP-1 promotes satiety and thereby facilitates body weight loss. Several GLP-1 receptor agonists (GLP-1RAs) are used successfully in the treatment of type 2 diabetes-including insulin-treated persons with type 2 diabetes. ${ }^{17}$ Based on their pharmacokinetic profiles, the GLP-1RAs can be divided into short-acting or long-acting compounds with important between-class differences.

In type 2 diabetes, long-acting compounds exert continuous insulinotropic and glucagonostatic effects. Therefore, they have a greater-and sustained-effect on fasting plasma glucose compared with the short-acting GLP-1RAs. Lowering of fasting plasma glucose is pivotal in insulin-treated type 2 diabetes, and long-acting-compound treatment therefore generally translates into better glycaemic control compared with short-acting GLP-1RAs. In contrast, treatment with short-acting GLP-1RAs exerts potent and sustained slowdown of gastric emptying with an effective lowering of postprandial plasma glucose excursions, an effect lost with long-acting GLP-1RAs due to tachyphylaxis. ${ }^{17}$ Thus, persons with adequately controlled fasting plasma glucose that are in need for postprandial glucose lowering to achieve glycaemic control will most likely benefit more from a short-acting GLP-1RA compared with a long-acting GLP-1RA. ${ }^{18}$ In contrast to the different, glucose-lowering effects of the different GLP-1RAs, the body weight-reducing effects of GLP-1RAs seem independent of their pharmacokinetic profile. ${ }^{17}$

GLP-1RAs provide a valuable treatment concept for persons with type 2 diabetes. Their insulin-independent effects, that is, glucose-dependent glucagon suppression (occurring only at plasma glucose concentrations above $4-5 \mathrm{mmol} / \mathrm{L}$ ), appetite reduction and deceleration of gastric emptying, make them interesting from a type-1-diabetes management perspective. The long-acting GLP-1RA, liraglutide, was previously examined in several randomised, double-blinded, placebo-controlled trials as add-on treatment in persons with type 1 diabetes. These studies indicated substantial reductions in body weight and total exogenous insulin dose and, in general, moderate improvements in glycaemic control, but at the expense of increased incidences of symptomatic hypoglycaemia and hyperglycaemia with ketosis in the two ADJUNCT studies. ${ }^{19-23}$

Importantly, the effect of short-acting compounds on postprandial glucose excursions may be of particular interest as several studies have shown a strong correlation between postprandial glucose control and glycated haemoglobin (HbAlc) in type 1 diabetes. ${ }^{18}$ However, no large controlled clinical trial evaluating the short-acting GLP-1RA treatment effect in type 1 diabetes has been reported. Smaller, mainly mechanistic, studies of exenatide, a short-acting GLP-1RA normally administered two times per day, have shown reductions in postprandial glucose excursions and insulin requirements $(0.17$ $1.19 \mathrm{U} / \mathrm{kg} /$ day $)$ together with weight loss $(2.8-4.5 \mathrm{~kg})$ and improved, or at least unaltered, glycaemic control. ${ }^{24-26}$ The main mechanisms for these effects seem to involve deceleration of gastric emptying, ${ }^{27-29}$ and possibly reduced postprandial glucagon secretion. ${ }^{30} 31$ Importantly, exenatide given two times per day did not decrease the glucagon response during a hypoglycaemic clamp after 4 weeks of treatment, ${ }^{32}$ indicating that exenatide's blood glucose-lowering effects do not compromise the main counter-regulatory effects during hypoglycaemia.

\section{Hypothesis}

We hypothesise that add-on therapy of exenatide $10 \mu \mathrm{g}$ three times per day at main meals to basal-bolus insulin therapy in normal to overweight/obese persons with type 1 diabetes with inadequate glycaemic control (HbA1c between 58 and $86 \mathrm{mmol} / \mathrm{mol}$ ) will reduce insulin requirements, glycaemic excursions and body weight and improve glycaemic control without increasing the risk of hypoglycaemia.

\section{Objectives and endpoints}

The overall objective of the present study is to evaluate the safety and efficacy of the short-acting GLP-1RA, exenatide, administered three times per day (before each main meal) as add-on therapy to standard basal-bolus insulin regimen in persons with type 1 diabetes. The primary endpoint is change in HbAlc after 26 weeks of treatment compared with placebo. Secondary endpoints include changes in glycaemic excursions; insulin dose; hypoglycaemic and adverse events; body weight, lean body mass, fat mass; dietary patterns; quality of life and treatment satisfaction; cardiovascular-disease risk profile; metabolomics; and arginine-tested plasma glucose, glucagon and C-peptide levels (box 1).

\section{Trial design}

The MAG1C trial (Meal-time Administration of Exenatide for Glycaemic Control in Type 1 Diabetes Cases: a randomised, placebo-controlled trial) is a 26weekinvestigator-initiated, two-armed, parallel group, randomised, double-blinded and placebo-controlled study.

\section{METHODS AND ANALYSIS}

In total, 100 persons with type 1 diabetes on basal-bolus insulin therapy will be randomised in a 1:1 ratio to either meal-time exenatide $10 \mu \mathrm{g}$ three times per day or placebo as add-on therapy to regular insulin treatment. 


\section{Box 1 Primary and secondary endpoints}

Primary endpoint

$>\mathrm{HbA1C}$

Secondary endpoints

- CGM: Glycaemic variability and time spent in hypoglycaemia, near normoglycaemia and hyperglycaemia

- Insulin dose

- Hypoglycaemic events

Body weight

$\rightarrow$ BMI

- Body composition (hip:waist ratio)

- DXA scan: Lean body mass and fat mass composition

- Fasting plasma glucose

Dietary patterns

- Arginine test: Pre/poststimulatory levels of glucagon, C-peptide and glucose

- Cardiovascular disease risk profile: Cholesterol levels, biomarkers, blood pressure and heart rate

Q Quality of life and treatment satisfaction

- Adverse events

BMI, body mass index; CGM, continuous glucose monitoring; DXA, dual-energy X-ray absorptiometry; HbA1c, glycated haemoglobin.

A study-independent person will use a computer-generated randomisation list for treatment allocation. Data will be stored in paper-based case report files (CRF). Double data entry into a digital database with range checks for data values will be used. In case of emergency, unblinding will be made on an individual basis not affecting other study participants. All data will be pseudoanonymised.

\section{Study population}

Study participants will be recruited from outpatient clinics in the Capital Region of Denmark. All recruited participants meeting the eligibility criteria at screening will be enrolled in the study and treated for the following 26 weeks at the Steno Diabetes Center Copenhagen, Gentofte, Denmark (box 2).

\section{Trial visits and examinations}

Study participants will be provided with written and oral information by the investigator prior to obtaining written informed consent at Steno Diabetes Center Copenhagen. At screening (visit 0), information on demography, medical history, smoking/drinking status and concomitant medication will be obtained. Further, a physical assessment will be made including heart rate, blood pressure, body weight, hip:waist ratio and electrocardiography together with blood samples and urine tests (box 3). Six-day continuous glucose monitoring (CGM), together with a 3-day diet recording, will be made before randomisation (visit 1), at week 4 (visit 2) and at end of treatment (visit 4). Participants not familiar with carbohydrate counting will be offered a standard course before entering the study. Blood samples and urine tests will be taken during the trial (box 3). An arginine test and a dual-energy X-ray absorptiometry (DXA) scan will be made at randomisation (visit 1) and at end of treatment (visit 4)

\section{Box 2 Eligibility criteria}

Inclusion criteria

- Type 1 diabetes according to WHO criteria with duration of $\geq 1$ year

- Age $\geq 18$ years

- $\mathrm{BMI}>22.0 \mathrm{~kg} / \mathrm{m}^{2}$

- $\mathrm{HbA} 1 \mathrm{C}>7.5 \%$ and $<10.0 \%$ at visit 0 (screening)

- Able to count carbohydrates

- Able to understand the written patient information and to give informed consent

Exclusion criteria

- Insulin pump treatment

- Hypoglycaemia unawareness (inability to register low blood glucose)

- Diabetic gastroparesis

- Compromised kidney function (eGFR $<60 \mathrm{~mL} / \mathrm{min} / 1.73 \mathrm{~m}^{2}$, dialysis or kidney transplantation)

- Liver disease with elevated plasma alanine aminotransferase $>$ three times the upper limit of normal (measured at visit 0 with the possibility of one repeat analysis within a week, and the last measured value as being conclusive)

- History of acute and/or chronic pancreatitis

- Subjects with personal or family history of medullary carcinoma or MEN syndrome

- Inflammatory bowel disease

- Cancer, unless in complete remission for $>5$ years

- Proliferative retinopathy

- Other concomitant disease or treatment that according to the investigator's assessment makes the patient unsuitable for study participation

- Alcohol/drug abuse

- Fertile women not using chemical (tablet/pill, depot injection of progesterone, subdermal gestagen implantation, hormonal vaginal ring or transdermal hormonal patch) or mechanical (spirals) contraceptives

- Pregnant or nursing women

- Known or suspected hypersensitivity to trial product or related products

- Receipt of an investigational drug within 30 days prior to visit 0

- Simultaneous participation in any other clinical intervention trial Withdrawal criteria

- In case of pregnancy (or desire for pregnancy), female subjects are withdrawn

- Lack of compliance to any of the important study procedures in the discretion of the investigator

- Onset of any disorder considered to compromise the safety by participating in the study

- Unacceptable adverse effects in the discretion of the investigator

- Withdrawal on participants request will be accepted at any time without further justification

BMI, body mass index; eGFR, estimated glomerular filtration rate; HbA1c, glycated haemoglobin; MEN, multiple endocrine neoplasia types 1 and 2.

(box 3). Insulin doses will be adjusted by the investigator or qualified study personnel during the trial at study visits based on seven-point plasma glucose profiles, CGM and HbA1c. Blood glucose treatment targets will be based on international guidelines, ${ }^{9}$ that is, preprandial values of $4-7 \mathrm{mmol} / \mathrm{L}$ and postprandial values $<10 \mathrm{mmol} / \mathrm{L}$. Following randomisation, changes in insulin types are not allowed. The study participants will be asked to fill 


\section{Box 3 Blood samples}

Screening and control visits

- Blood haemoglobin, leucocytes, thrombocytes, plasma glucose, potassium, sodium, creatinine, TSH, cholesterol, triglycerides, ALT, AST, amylase, lipase, serum albumin, total serum-ketones, beta-hydroxybutyrate and acetoacetate

Biobank

- CVD markers: HsCRP, pro-BNP

Bone markers: CTX, P1NP, sclerostin, osteocalcin

Inflammation markers: IL-2, IL-6, TNF- $\alpha$

- Urine albumin:creatinine ratio, hCG

Arginine test

- Glucagon, C-peptide, plasma glucose

ALT, alanine aminotransferase; AST, aspartate aminotransferase; CTX, C-terminal telopeptide of type 1 ; CVD, cardiovascular disease; $\mathrm{hCG}$, human choriongonadotropin; hsCRP, high-sensitivity $\mathrm{C}$ reactive protein; IL-2, interleukin-2; IL-6, interleukin-6, P1NP, serum type 1 procollagen N-terminal; pro-BNP, prohormone brain natriuretic peptide; TNF- $\alpha$, tumour necrosis factoralpha; TSH, thyroid-stimulating hormone. out questionnaires on quality of life (The Audit of Diabetes-Dependent Quality of Life) and Diabetes Treatment Satisfactory Questionnaire: status (DTSQs) and change version (DTSQc). ${ }^{33} 34$ Information on adverse events, current medication, basal-bolus insulin dose, hypoglycaemic events and consultation blood pressure and heart rate will be recorded at all visits. Body weight and hip:waist ratio will be measured as well, except on visit 3 . Between-visit telephone contacts will be made to ensure the study participants' safety and compliance together with evaluation of insulin treatment. Further, the study participants will be instructed to contact the study team if any insulin-dosing or glucose control problems occur. All contacts will be recorded in the CRF (figure 1 and table 1). To further attenuate the risk of hypoglycaemia, no insulin is taken on the visit 1 study day and plasma glucose is measured before administration of the first dose

Figure 1 Flowchart.

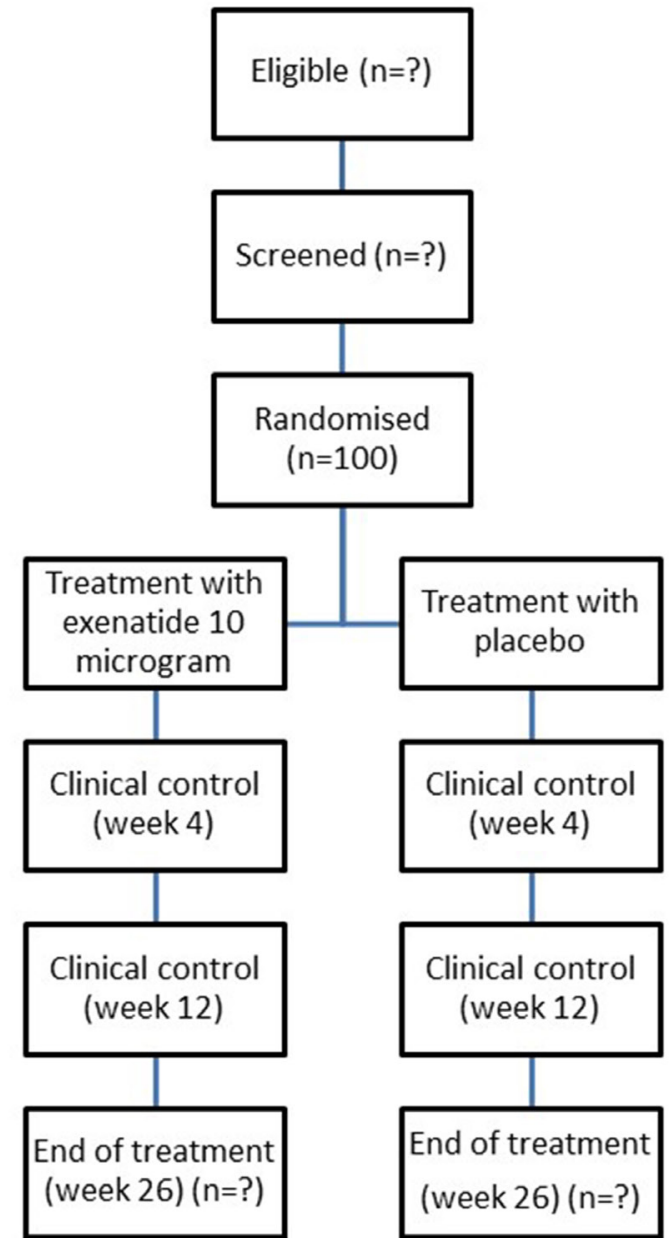




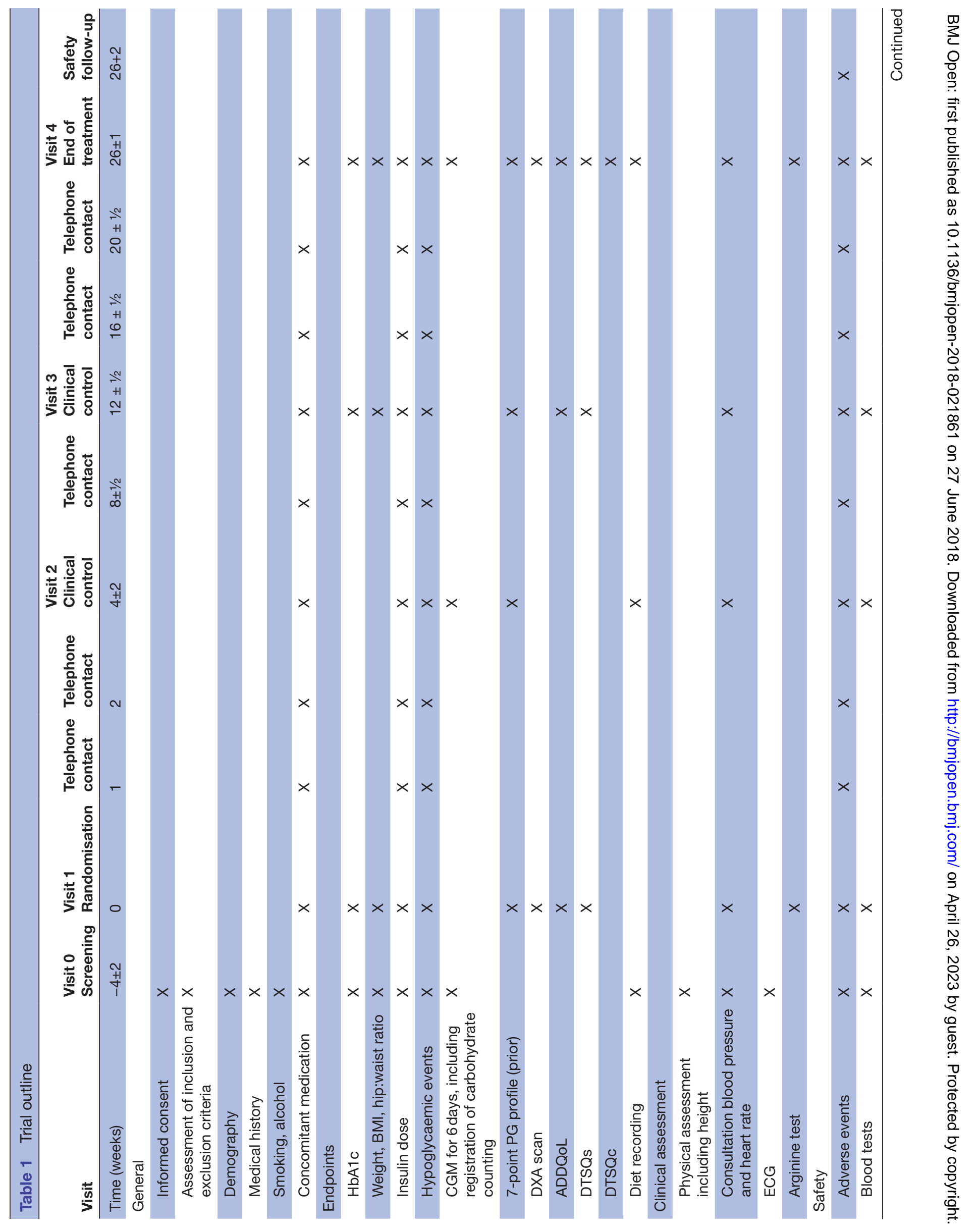




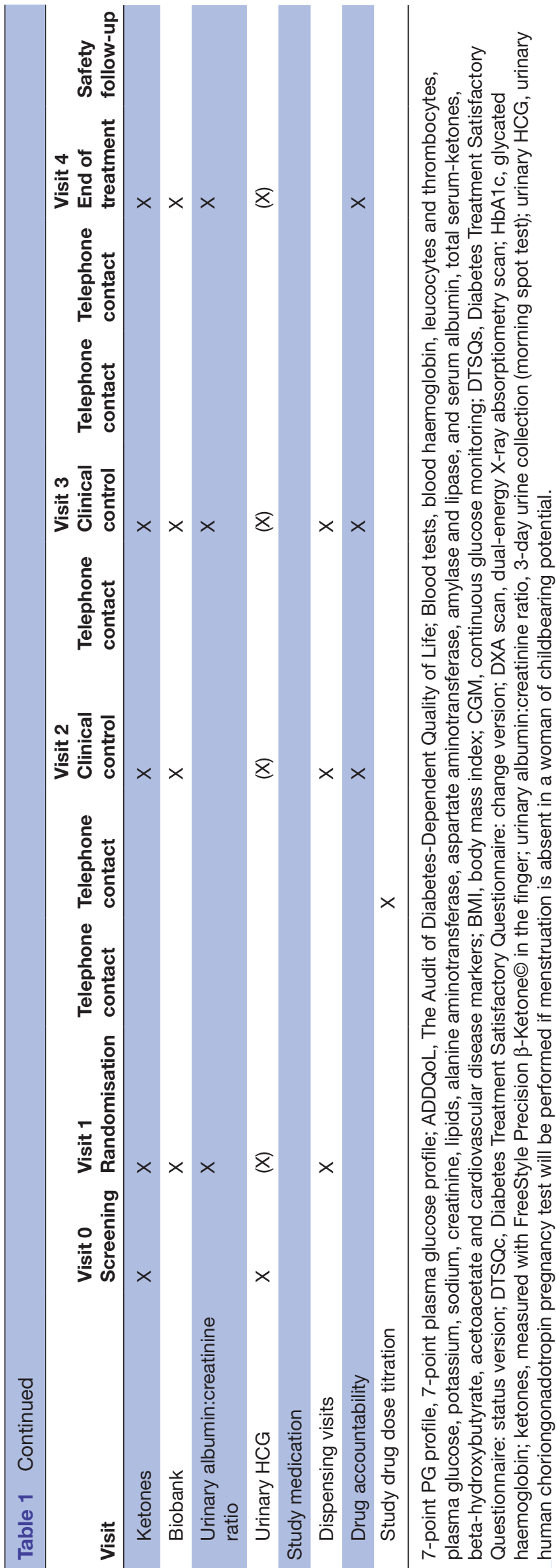

of investigational product and $30 \mathrm{~min}$ following ingestion of a standardised meal. Next, telephone contacts are made 1 and 2 weeks after randomisation with careful instruction on reporting any hypoglycaemic and hyperglycaemic events. Finally, the investigational product is started at $5 \mu \mathrm{g}$ and escalated to $10 \mu \mathrm{g}$ following telephone contact 2, if tolerated. All contacts will be recorded in the CRF (figure 1 and table 1). Finally to ensure compliance, used investigational product cartridges will be collected at study visit.

A substudy on the role of the microbial gut flora, approved by the Danish regulatory authorities and voluntary to participate in, involving the collection of faecal specimens at each study visit will also be conducted.

\section{Patient and public involvement}

The MAG1C study aims to attenuate intensive insulin treatment side effects: hypoglycaemia and weight gain. Further, our study drug regimen will, hopefully, make it easier to control blood glucose excursions on a daily basis. We evaluate study participant treatment satisfaction and quality of life through questionnaires during the study period to make sure our results benefit persons with type 1 diabetes. Study participants will be informed of our results in layman's terms as well as their individual exenatide/placebo assignment by letters following publication. Finally, during the study protocol write-up, a colleague of ours with type 1 diabetes read and commented on the final protocol draft.

\section{Intervention}

Name: Byetta (exenatide) or matching placebo.

Pharmaceutical form: exenatide $0.25 \mathrm{mg} / \mathrm{mL}, 3 \mathrm{~mL}$ cartridges in a reusable Ypsopen, for subcutaneous injection. Placebo, $3 \mathrm{~mL}$ cartridges in a reusable Ypsopen, for subcutaneous injection.

Pharmaceutical dosage: To minimise the side effect risk, exenatide dose, or placebo, will be increased from initial $5 \mu \mathrm{g}$ three times per day to full dosage, $10 \mu \mathrm{g}$ three times per day, 2 weeks after randomisation. The injection must occur within 1 hour before the main meals. Dose increments can be titrated based on the individual study participant's study drug tolerance, to a minimum of $5 \mu \mathrm{g}$ three times per day 3 months after randomisation. If not possible at this time, the participant will be withdrawn from the study.

Side effects: Common side effects $(1 \%-10 \%)$ include nausea, vomiting, diarrhoea, hypoglycaemia and headache. Study participants will be carefully instructed to avoid dehydration if gastrointestinal side effects occur.

Shipping and packing: All study medication will be produced, blinded, packed and delivered by AstraZeneca, the producer of Byetta.

\section{Sample size}

To be able to detect a difference in change in HbAlc (primary outcome) between study arms of $6 \mathrm{mmol} / \mathrm{mol}$ with $80 \%$ power, a $5 \%$ significance level and a presumed 
$9 \mathrm{mmol} / \mathrm{mol} \mathrm{SD}, 42$ persons should be included in each study arm (two-sided test). To allow for a $20 \%$ dropout rate, 100 persons in total will be included in the study: 50 in each study arm. The sample size calculation is based on data from a similar study on the GLP-1RA, liraglutide. ${ }^{21}$ Withdrawn study participants will not be replaced.

\section{Data analysis}

The per-protocol study population includes all participants who complete the study with a documented, valid baseline and end-of-treatment assessment of the primary endpoint without any major protocol violations. In case of dropout, last observation is carried forward. The intention-to-treat population includes all randomised persons. Primary-endpoint analysis will be based on the per-protocol population. Absolute differences and adjusted mean changes between groups, together with $95 \% \mathrm{CI}$, will be reported. The efficacy analysis will be carried out with a linear mixed-effect model with visit, treatment and their interaction as fixed factors and a random intercept on the person level. Variables that are normally distributed will be presented as mean $\pm \mathrm{SD}$ or $\mathrm{SE}$ of the mean. In case of non-normal distribution, non-parametric statistics and $\log$ transformation will be used. A two-tailed $p$ value $\leq 0.05$ will be considered statistically significant. Additional analyses will be made from the intention-to-treat population to assess the validity of the per-protocol population conclusions if loss of follow-up occurs. These calculations will include duration in study and reason for discontinuation.

Following completion of last patient last visit, unblinding will be made in two steps. During data analysis, unblinding will be made on group level, that is, participants are assigned to groups 1 and 2. After the prespecified data analysis is completed, the specific treatment group will be revealed.

\section{ETHICS AND DISSEMINATION}

We expect the present study to generate important information about the use of short-acting GLP-1RAs as add-on therapy to insulin in persons with type 1 diabetes. We expect to be able to answer two questions relevant for numerous persons worldwide: Will meal-time exenatide $10 \mu \mathrm{g}$ added three times per day (at each main meal) to regular insulin therapy (1) improve postprandial glycaemic excursions and (2) provide improved, long-term glycaemic control measured as HbA1c and glycaemic variability?

During the study, a physician will follow each participant with careful evaluation of insulin treatment with glycaemic optimisation and study drug safety and efficacy. This is expected to lower the adverse event risk. Exenatide is approved for the treatment of type 2 diabetes by the European Medicines Agency and by the US Food and Drug Administration. Prior studies have shown limited side effects such as nausea, vomiting, hypoglycaemia and headache. Nausea and vomiting, generally transient, usually occur within 3 weeks after treatment initiation. They can be minimised by gradual dose titration, as planned in this study. The hypoglycaemia risk is reduced by insulin dose reduction at study start and by instructing participants in careful blood glucose monitoring. Few cases of acute pancreatitis have been reported in persons with type 2 diabetes using exenatide, but the incidence was similar to the type 2 diabetes background population. Overall, the risk of side effects in this study is expected to be modest. Arginine injection is a well-validated, safe method to evaluate pancreatic alpha and beta cell function, but potentially associated with transient mild flushing, nausea and metallic taste. Vein puncture may cause a short pain, risk of a small haematoma and a minimal risk of puncture site infection. In total, $400 \mathrm{~mL}$ blood and $80 \mathrm{~mL}$ of urine per person will be collected throughout the study. At the two DXA scans, participants will be exposed to weak X-ray radiation (less than $1 \mathrm{mSv}$ in total). For comparison, the background radiation in Denmark is about $3 \mathrm{mSv}$ per year. The risk of complications, or adverse events, is negligible for all other planned study procedures.

Data will be processed and merged into one or more scientific articles and published in accordance with the CONSORT 2010 statement in international, peer-reviewed scientific journals and presented at national and international scientific meetings. Positive, negative and inconclusive results together with statistical method will be published as soon as scientifically justifiable. AstraZeneca commented on the study design but will have no influence on trial conduction, data analysis, interpretation or publication. All data are owned by the authors, who all have full data access.

\section{Study approval}

The MAG1C trial is approved with a current, approved (15 February 2018) study protocol V.1.6. It is registered at ClinicalTrials.gov (NCT03017352). The study will be conducted under the surveillance and guidance of the good clinical practice (GCP) unit at Copenhagen University Hospital Bispebjerg in accordance with the ICH-GCP guidelines and the Helsinki Declaration. The study commenced in January 2017 and is expected to be reported in 2019. Presently (May 2018), 58 study participants have been included in the MAG1C study.

Acknowledgements We would like to thank Andreas Brønden, MD PhD, as our patient adviser for reading and commenting on the final draft of the study protocol.

Contributors NJJ, TFD, AL, TV, HUA and FKK conceived and designed the study. FKK is guarantor of the study and sponsors the trial. NJJ drafted the manuscript and all authors have contributed to the revision of the manuscript and read and approved the final version of the manuscript.

Funding This investigator-initiated research was conducted with support from AstraZeneca.

Competing interests NJJ and TV have no competing interests. TFD has received research support from Novo Nordisk and AstraZeneca, and has received lecture fees from Novo Nordisk. AL has received lecture fees from Novo Nordisk, Boehringer Ingelheim and Eli Lilly. HUA owns stocks in Novo Nordisk and serves in advisory boards for Novo Nordisk and Astra Zeneca. FKK has served on scientific advisory panels and/or speaker's bureaus for, served as a consultant to and/or received research support from Amgen, AstraZeneca, 
Boehringer Ingelheim, Eli Lilly, Gubra, MSD/Merck, Novo Nordisk, Sanofi and Zealand Pharma.

\section{Patient consent Not required.}

Ethics approval Danish Medicines Authority (Eudract-nr.: 2016-00136592); Regional Scientific-Ethics Committee of the Capital Region of Denmark (H-16034515); Data Protection Agency (2012-58-0004)

Provenance and peer review Not commissioned; externally peer reviewed.

Data sharing statement Data will be processed and merged into one or more scientific articles and published in accordance with the CONSORT 2010 statement in international, peer-reviewed scientific journals and presented at national and international scientific meetings. Positive, negative and inconclusive results together with statistical method will be published as soon as scientifically justifiable. AstraZeneca commented on the study design but will have no influence on trial conduction, data analysis, interpretation or publication. All data are owned by the authors, who all have full data access.

Open access This is an open access article distributed in accordance with the Creative Commons Attribution Non Commercial (CC BY-NC 4.0) license, which permits others to distribute, remix, adapt, build upon this work non-commercially, and license their derivative works on different terms, provided the original work is properly cited and the use is non-commercial. See: http://creativecommons.org/ licenses/by-nc/4.0/

(c) Article author(s) (or their employer(s) unless otherwise stated in the text of the article) 2018. All rights reserved. No commercial use is permitted unless otherwise expressly granted.

\section{REFERENCES}

1. IDF. IDF diabetes atlas. 8th Edn: IDF. (accessed 19 Dec 2017).

2. Atkinson MA, Eisenbarth GS, Michels AW. Type 1 diabetes. The Lancet 2014;383:69-82.

3. Miller KM, Foster NC, Beck RW, et al. Current state of type 1 diabetes treatment in the U.S.: updated data from the T1D Exchange clinic registry. Diabetes Care 2015;38:971-8.

4. McKnight JA, Wild SH, Lamb MJE, et al. Glycaemic control of Type 1 diabetes in clinical practice early in the 21 st century: an international comparison. Diabetic Medicine 2015;32:1036-50.

5. Weinstock RS, Schütz-Fuhrmann I, Connor CG, et al. Type 1 diabetes in older adults: Comparing treatments and chronic complications in the United States T1D Exchange and the German/ Austrian DPV registries. Diabetes Res Clin Pract 2016;122:28-37.

6. Conway B, Miller RG, Costacou T, et al. Temporal patterns in overweight and obesity in Type 1 diabetes. Diabet Med 2010;27:398-404.

7. Nathan DM, Genuth S, Lachin J, et al. The effect of intensive treatment of diabetes on the development and progression of longterm complications in insulin-dependent diabetes mellitus. $N$ Engl J Med 1993;329:977-86.

8. Nathan DM, Cleary PA, Backlund JY, et al. Intensive diabetes treatment and cardiovascular disease in patients with type 1 diabetes. N Engl J Med 2005;353:2643-53.

9. American Diabetes Association. Standards of medical care in diabetes--2014. Diabetes Care 2014;37(Suppl 1):S14-80.

10. Russell-Jones D, Khan R. Insulin-associated weight gain in diabetes-causes, effects and coping strategies. Diabetes Obes Metab 2007:9:799-812.

11. Cryer PE. Hypoglycemia: still the limiting factor in the glycemic management of diabetes. Endocr Pract 2008;14:750-6.

12. Cryer PE, Davis SN, Shamoon H. Hypoglycemia in diabetes. Diabetes Care 2003;26:1902-12.

13. Lund A, Knop FK. Worry vs. knowledge about treatment-associated hypoglycaemia and weight gain in type 2 diabetic patients on metformin and/or sulphonylurea. Curr Med Res Opin 2012;28:731-6.
14. Must A, Spadano J, Coakley EH, et al. The disease burden associated with overweight and obesity. JAMA 1999;282:1523-9.

15. Frandsen CS, Dejgaard TF, Madsbad S. Non-insulin drugs to treat hyperglycaemia in type 1 diabetes mellitus. Lancet Diabetes Endocrinol 2016;4:766-80.

16. Nauck MA, Kemmeries G, Holst JJ, et al. Rapid tachyphylaxis of the glucagon-like peptide 1-induced deceleration of gastric emptying in humans. Diabetes 2011;60:1561-5.

17. Lund A, Knop FK, Vilsbøll T. Glucagon-like peptide-1 receptor agonists for the treatment of type 2 diabetes: differences and similarities. Eur J Intern Med 2014;25:407-14.

18. American Diabetes Association. Postprandial blood glucose. American Diabetes Association. Diabetes Care 2001;24:775-8.

19. Mathieu C, Zinman B, Hemmingsson JU, et al. Efficacy and safety of liraglutide added to insulin treatment in type 1 diabetes: the adjunct one treat-to-target randomized trial. Diabetes Care 2016;39:1702-10.

20. Ahrén B, Hirsch IB, Pieber TR, et al. Efficacy and safety of liraglutide added to capped insulin treatment in subjects with type 1 diabetes: the adjunct two randomized trial. Diabetes Care 2016;39:1693-701.

21. Dejgaard TF, Frandsen CS, Hansen TS, et al. Efficacy and safety of liraglutide for overweight adult patients with type 1 diabetes and insufficient glycaemic control (Lira-1): a randomised, doubleblind, placebo-controlled trial. Lancet Diabetes Endocrinol 2016;4:221-32.

22. Frandsen CS, Dejgaard TF, Holst JJ, et al. Twelve-Week treatment with liraglutide as add-on to insulin in normal-weight patients with poorly controlled type 1 diabetes: a randomized, placebo-controlled, double-blind parallel study. Diabetes Care 2015;38:2250-7.

23. D T, F CS, S S, et al. Efficacy and safety of liraglutide in insulin pump treated people with type 1 diabetes: The lira pump trial. Diabetologia (published online first Sep 2017).

24. Rother KI, Spain LM, Wesley RA, et al. Effects of exenatide alone and in combination with daclizumab on beta-cell function in longstanding type 1 diabetes. Diabetes Care 2009;32:2251-7.

25. Ghazi T, Rink L, Sherr JL, et al. Acute metabolic effects of exenatide in patients with type 1 diabetes with and without residual insulin to oral and intravenous glucose challenges. Diabetes Care 2014;37:210-6.

26. Sarkar G, Alattar M, Brown RJ, et al. Exenatide treatment for 6 months improves insulin sensitivity in adults with type 1 diabetes. Diabetes Care 2014;37:666-70.

27. Plummer MP, Jones KL, Cousins CE, et al. Hyperglycemia potentiates the slowing of gastric emptying induced by exogenous GLP-1. Diabetes Care 2015;38:1123-9.

28. Plummer MP, Jones KL, Annink CE, et al. Glucagon-like peptide 1 attenuates the acceleration of gastric emptying induced by hypoglycemia in healthy subjects. Diabetes Care 2014;37:1509-15.

29. Bharucha AE, Batey-Schaefer B, Cleary PA, et al. Delayed gastric emptying is associated with early and long-term hyperglycemia in type 1 diabetes mellitus. Gastroenterology 2015;149:330-9.

30. Kielgast $U$, Holst JJ, Madsbad S. Antidiabetic actions of endogenous and exogenous GLP-1 in type 1 diabetic patients with and without residual $\beta$-cell function. Diabetes 2011;60:1599-607.

31. Kramer CK, Borgoño CA, Van Nostrand $P$, et al. Glucagon response to oral glucose challenge in type 1 diabetes: lack of impact of euglycemia. Diabetes Care 2014;37:1076-82.

32. Jiang LL, Wang SQ, Ding B, et al. The effects of add-on exenatide to insulin on glycemic variability and hypoglycemia in patients with type 1 diabetes mellitus. J Endocrinol Invest 2018;41.

33. Bradley C, Todd C, Gorton T, et al. The development of an individualized questionnaire measure of perceived impact of diabetes on quality of life: the ADDQoL. Qual Life Res 1999;8:79-91.

34. Bradley C, Plowright R, Stewart J, et al. The Diabetes Treatment Satisfaction Questionnaire change version (DTSQc) evaluated in insulin glargine trials shows greater responsiveness to improvements than the original DTSQ. Health Qual Life Outcomes 2007;5:57. 